\title{
Cryoanesthesia as a Supplementary Aid to the Mandibular Anesthetic Technique in Endodontics: A Preliminary Experience
}

\author{
Pedro Mejía Fernández ${ }^{1}$, Martha Rebolledo Cobos ${ }^{2 *}$ and María de los Ángeles Torres Barros ${ }^{1}$ \\ ${ }^{1}$ Universidad Metropolitana de Barranquilla, Colombia \\ ${ }^{2}$ Stomatologist and Oral Surgery Specialist, Colombia
}

\begin{abstract}
Objective: Describe the cryoanesthetic effect of the use of dry ice (solid Carbon Dioxide) spray in patients with Irreversible Pulpitis (IP) undergoing conventional endodontic treatment in lower molars.

Materials and Methods: This are an-in vivo quasi-experimental study with a convenience sample of 126 patients who met the criteria for selection. The clinical history was completed, obtaining socio-demographic characteristics, including the diagnosis of IP. After previously signing informed consent, patients were anesthetized applying the inferior alveolar anesthetic technique; after a period of five minutes, molars were completely isolated, then dry ice spray was applied for two seconds. Subsequently, a conventional access cavity was opened, applying intrapulpal anesthesia upon pulp exposure. For the purpose of measuring the pain experienced by the patients during access cavity opening and instrumentation procedures, the Heft-Parker modified visual analog scale (HPVAS) was used.
\end{abstract}

Results: $60 \%$ of the subjects (sample) were female, the average age range was between 53 and 63 years. The tooth most frequently affected by IP was 46 . According to HPVAS, $94.4 \%$ of patients reported feeling no pain, gender had no influence on the outcome of HPVAS. Based on the odds ratio related to Chi2 at older age, there is a $1.6 \%$ probability of success per year. Age showed a $P$ value of less than 0.05 . The relation between gender and the affected tooth did not show a statistically significant difference.

Conclusion: Cryoanesthesia was successful as a supplementary aid to mandibular anesthesia in the endodontic treatment of lower molars.

KEYWORDS: Cryoanesthesia; Cold; Therapy; Analgesia; Anesthesia; Anesthetic technique; Mandibular anesthetic technique; Endodontics

ABBREVIATIONS: IP: Irreversible Pulpitis; HPVAS: Heft-Parker Visual Analogue Scale; EPT: Electrical Pulp Tests

\section{INTRODUCTION}

Treatments in which cold therapy is used in Dentistry may be referred to as physiotherapeutic alternatives applied in different ways ad with a variety of effects. Physiological and clinical evidence suggests that applying cold therapy reduces nerve conduction velocity, hemorrhage, edema and local inflammation [1].
Quick Response Code:

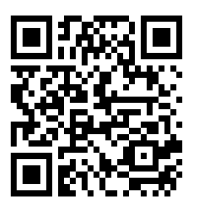

Address for correspondence: Martha Rebolledo Cobos, Metropolitan University, Faculty of Dentistry, Colombia

Received: November 21, 2019 Published: December 03, 2019

How to cite this article: Pedro MF, Martha RC, María de los ÁTB. Cryoanesthesia as a Supplementary Aid to the Mandibular Anesthetic Technique in Endodontics: A Preliminary Experience. 2019 - 1(3) OAJBS.ID.000123. DOI: 10.38125/OAJBS.000123 
The first physiological response of a tissue upon exposure to the cold is a drop in local temperature, which reduces cellular metabolism, causing cells to consume less oxygen and decreasing blood flow induced by vasoconstriction, thus limiting damage. Furthermore, cold exposure affects peripheral terminations of nerves by diminishing the threshold necessary to activate nociceptors and the velocity of painful nerve impulses. The main purpose of cold therapy is sedation, though it can also provide additional benefits such as analgesia, anti-inflammatory and circulatory effects in surgical procedures, amongst others, but in present day dentistry there is no published evidence of the use of cold therapy as an anesthetic aid [2]. In the last century, it was used as an anesthetic technique in dentistry, but due to the traumatic effect on the patient, the rudimentary aspect of the equipment available at the time, and the evolution of anesthetic techniques into injectable anesthetics, its use was discontinued; nevertheless, there were positive outcomes that justify the use of the term "Cryoanesthesia" [3].

Local anesthesia administration techniques are a fundamental aspect of professional dental practice, as optimum efficacy is required prior to starting a variety of treatments. Anesthetic administration procedures require the use of the appropriate technique and anesthetic substance in order to be effective. However, failure in the application of anesthesia may occur. Every Dentist must face anesthetic failure at some point in their careers; that is why professionals must be familiar with the associated etiology, and with the strategies for handling and preventing this situation [4,5]. The mandibular anesthetic technique, also known as inferior alveolar nerve block, is the most common technique in clinical dentistry, but also the one with the highest incidence of failure due to different factors that could be related to the operator, the patient, or those attributable to anatomic, pathological and psychological aspects, amongst others $[4,5]$.

Dry ice spray has been recently used in dentistry in a variety of commercial presentations, mostly as a differential diagnosis test in pulpal pathologies, temporarily anesthetizing the tooth involved, which might justify its use as a complementary aid in conventional anesthetic techniques [6]. At present, possible clinical applications of cold therapy as a cryoanesthetic agent have not been thoroughly explored, especially regarding the thermal effects in nerve impulse inhibition. In general terms, current evidence supports its use as a cryo-analgesic agent for the treatment of extra-oral swelling following an extraction, or as final irrigation into the root canal to reduce periodontal pain after endodontic treatment $[7,8]$.

In light of the abovementioned statements, the objective of this study is to describe the temporary anesthetic effect or Cryoanesthesia ensued by the application of dry ice spray and its effectiveness as a supplementary technique for inferior alveolar nerve block in patients diagnosed with irreversible pulpitis undergoing root canal treatment.

\section{METHODOLOGY}

An In vivo quasi-experimental study with a population of 400 patients endodontically diagnosed with irreversible pulpitis (IP) who underwent dental treatment at a health institution in the city of Barranquilla- Colombia, within a period of 15 months. The final sample consisted of 126 patients with no gender distinction, obtained through non-probability convenience sampling, who met the selection criteria: systemically healthy patients in optimum cognitive conditions, with no history of allergy to local anesthetics or other medicines, who had not priorly taken any medications that might alter the perception of pain in case it occurred, with first, second or third molars showing clinical and radiographic signs of IP, with absence of apical radiolucent injury in periapical $x$ ray, and a vital pulp at the moment of opening access cavity.

Ethical standards for health-related research were observed in accordance with the principles established in the World Medical Association Declaration of Helsinki and the scientific, technical and administrative standards for health research, Resolution No. 008430 of 1993 by the Department of Health - Colombia. The treatments were performed on adult patients at a minimum risk due to their non-invasive nature. All participants freely signed an informed consent and were given the possibility to abandon research at any moment. This research was approved by the institutional bio-ethics committee.

\section{PROCEDURE}

Initially, a data collection instrument was filled out, which in this occasion shall be referred to as Medical History, considering the socio-demographic characteristics of the subjects of study, such as: age, gender, IP diagnosis, and affected tooth.

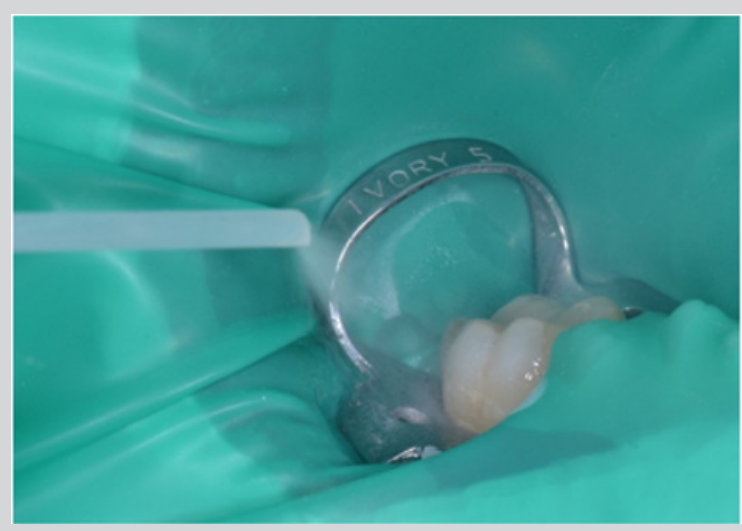

Figure 1: Application of cold spray for two seconds.

Once the patients were diagnosed and selected, inferior alveolar nerve block was performed, using double direct anesthesia of the spine of Spix with a 30-gauge short needle and lidocaine carpules with epinephrine $\AA_{1} 1.8 \mathrm{ml} 2 \%$ (Roxicaina ${ }^{\circledR}$ Ropsohn Therapeutics
Col). Five minutes after the administration of anesthesia, the tooth was fully isolated and then directly sprayed with the nozzle of Roeko Endo-Frost® cold spray (Coltene /Whaleden Inc USA) for two seconds (Figure 1). 
Three applications of two seconds each were sprayed in the tooth surfaces: occlusal, vestibular and then lingual. Next, conventional access cavities were opened with a $\mathrm{N}^{\circ} 2$ diamond bur ( ) to remove enamel or restorative material; intrapulpal anesthetic was injected upon exposure (lidocaine with epinephrine ${ }^{\circledR} 1.8 \mathrm{ml}$ $2 \%$ Roxicaina ${ }^{\circledR}$ Ropsohn Therapeutics Col) with a $\mathrm{N}^{\circ} 300.5 \mathrm{ml}$ needle, and access was continued with a $\mathrm{N}^{\circ} 4$ carbide bur. Finally, conductometry was measured and root canal instrumentation was initiated. To measure the pain experienced by patients during access cavity opening and instrumentation of root canal, a modified Heft-Parker visual analogue scale was used (HPVAS) [9]. The scale was divided into four categories: painless with a corresponding value of 0 , mild pain with a value of 1 , moderate pain with a value of 2 and severe pain with a corresponding value of 3 . Patients were instructed to raise their hands in the event of experiencing pain to register all findings.

Access cavity opening and instrumentation were defined as "Within pulpal space" and "Root canal instrumentation" and success was defined as absence of pain or mild pain during Access opening or instrumentation. Final data were collected in a Microsoft Excel 2017 file, and then initially analyzed by means of descriptive statistics: percentages were determined, and results were portrayed in graphs. The information was then processed in a statistic and graph analysis software called Stat graphics calculating the median within a confidence interval for the comparison of two dependent means (paired data).

\section{RESULTS}

Out of the 126 treated patients, $75(60 \%)$ were female and 51 $(40 \%)$ were male. With regards to age, 3 patients were in the range between 13-23 years, 7 between 23-33 years, 20 of them between 33-43, 24 between 43-53, 45 between 53-63, 22 between 63-73, 4 between $73-83$ and 1 between $83-93$ years. Therefore, we may conclude that most of the patients were in the age range between 53-63 years of age.

From these 126 cases, in 24 of them (19\%) the affected tooth was 36 , in 26 cases (21\%) the affected tooth was 37 , in 5 cases (4\%) the affected tooth was 38 , in 38 cases $(30 \%)$ the affected tooth was 46 , in 28 of the cases (22\%) the tooth was 47, in $5(4 \%)$ cases the treated tooth was 48 . Thus, the most treated tooth was 46 with a $30 \%$ frequency.

The percentage of patients who reported mild pain after receiving the new treatment was $94.44 \%$. From the total patient population, estimates are based on a 95\% interval for the ratio, which means that the actual percentage of patients reporting mild pain was between $88.8 \%$ and $97.7 \%$.

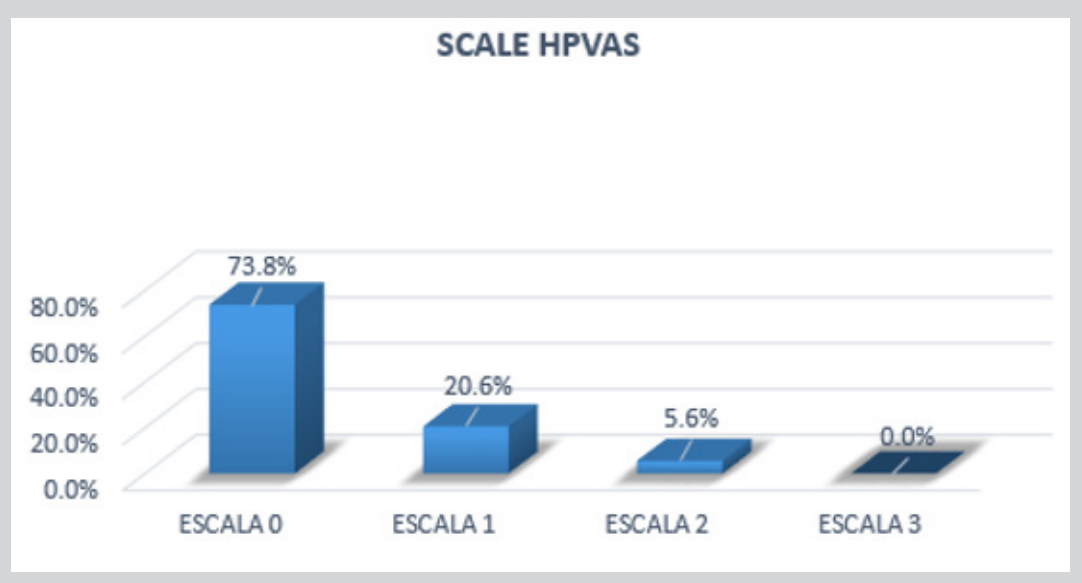

Figure 2: Percentage of distribution according to HPVAS.

Out of the 126 evaluations, according to HPVAS, 93 patients reported a painless procedure (0), adding up to $73.8 \%$, followed by 26 patients that perceived mild pain (1) with a 20.6\%, 7 described moderate pain (2) with an incidence of $5.6 \%$ and none reported severe pain (Figure 2). Consequently, we may conclude that most of the patients treated throughout this research perceived no pain during the procedure. In this study, success is considered as the absence of pain or the presence of mild pain. These two most frequent outcomes add up to a total success rate of $94.4 \%$.

Table 1: Test of independence regarding the relation between gender and the HPVAS results.

\begin{tabular}{|c|c|c|c|}
\hline Test & Statistic & G1 & P-Value* \\
\hline Chi-Square & 4.203 & 2 & 0.1223 \\
\hline
\end{tabular}

${ }^{*} P>0,05$ which indicates there is no significant difference regarding gender

Regarding the relation between gender and HPVAS outcome, the $p$ value in the chi Square test was evidently above 0.05 , from which may be concluded that gender has no influence on the HPVAS outcome after the application of cold spray in the subjects (Table 1).

A logistic regression model was used to establish the relation between age, gender and tooth with total absence of pain ( 0 or 1$)$ or with presence of pain (2 or 3) after applying cold spray, resulting in an age statistic value below 0.05 with a confidence interval equal to $95 \%$ (Table 2); (Figure 3). It is evident that at older age, the higher the probability of a positive result. At 25 years of age or older, approximately, the probability of a positive result surpasses $80 \%$ and is maintained then on. Based on the odds ratio related to $\mathrm{Chi}^{2}$ at older age, there is a $1.6 \%$ of probability of success per year of age (Figure 3).

Table 2: Success in relation to age, gender and tooth.

\begin{tabular}{|c|c|c|c|}
\hline Factor & Chi-Square & Gl & P-Value \\
\hline Age & 5.8045 & 1 & 0.0160 \\
\hline Gender & 0.0295325 & 1 & 0.8636 \\
\hline Tooth & 3.68718 & 5 & 0.5953 \\
\hline
\end{tabular}


Gráfica del Modelo Ajustado

con intervalos de confianza del $95,0 \%$

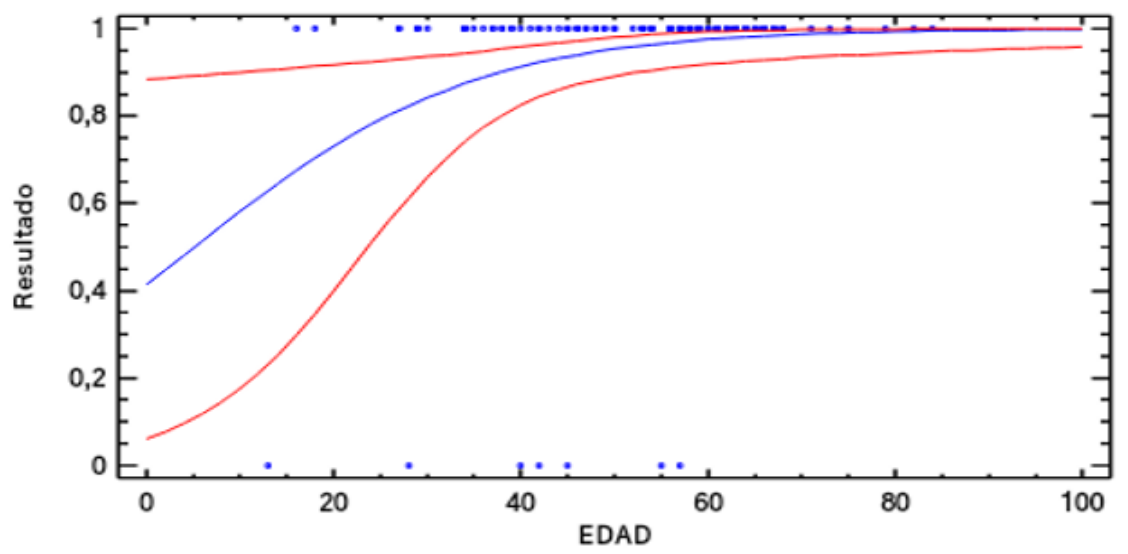

Figure 3: Gender and outcome ratio.

Beginning at approximately 25 years of age, the probability of a positive result is estimated to surpass $80 \%$ and is maintained then on.

\section{DISCUSSION}

The subject of pain relief, reduction or eradication through cryo-therapy in dental treatments is not new. Studies have proven that Oudry [10] in 1863 used a refrigerating machine prior to extractions. In 1937, Chaydeane and Black took the basis left by Oudry [10], which led them to experimenting with dogs and two human subjects. They used small hollow applicators firmly held against gum tissue on root level, which dropped the temperature to $33^{\circ} \mathrm{F}$. Using an electrical pulp tester, analgesia was found to occur in less than one minute [3].

In a preliminary study conducted by the Navy Medical Research Institute, National Navy Medical Center, Bethesda, Md histological research revealed that continuous cold exposure in localized areas of dogs' mandibles down to $1-2^{\circ} \mathrm{C}$ for two hours did not produce pathological alterations in gums, teeth nor surrounding structures [3].

Considering the results obtained in dogs, trials for the efficacy of cold therapy as local anesthetics in dental operatory procedures in human subjects were conducted. Preliminary results were encouraging in twenty-two opened access cavities, fifteen of which occurred in the complete absence of pain, whilst seven experienced mild pain. Similar results were found in clinical trials conducted in nine patients of the Dental and Oral Surgery School of the University of Columbia [3].

In 2015, Vera and cols re-introduce the term Cryotherapy with dental applications in their research [7]. Vera suggests a new methodology for intra-root canal cryotherapy based on the antiinflammatory effect of cold temperature. The specific objective of a first in vitro study was to determine whether external temperature in the 4 apical millimeters of the root canal could be reduced after continuously spraying cold saline solution $\left(2.5^{\circ} \mathrm{C}\right)$, maintained for at least 4 minutes to decrease post-operatory pain in Endodontics. In 2018, Vera conducts a clinical study with positive post-surgical antiinflammatory results. This process of diffusing cold temperature through the dentin and maintaining it for a period of 4 minutes is what accounts for the success of cryoanesthesia in our study [2].
Hsiao-WU GW assessed the success rate of mandibular anesthesia testing anesthetized molars with cold temperature. This proved that cases with a negative response to cold testing have $80 \%$ more probability of a painless treatment. However, the role that hypothermia may have played in the test was never considered [6].

Authors such as Candas and cols prove that intra canal, intra oral and extra oral cryotherapy reduce post-operatory pain, and therefore medicine intake as well [8].

At present, cryotherapy in endodontics is used in diagnostic testing by means of an easy, simple and available device: Endo ice ${ }^{\circledR}$ o Endofrost $\AA$ is an effective tool in diagnosing pulpal vitality, which reaches very low temperatures, even lower than those produced by dry ice and ethyl chloride [6].

This pulpal diagnostic method produces contraction of intra tubular dentin fluid, which prompts the fluid out of its tubular walls resulting in hydrodynamic forces that press the nerve fibers inside the pulp; this causes acute pain that will last throughout the whole clinical trial. A positive response indicates pulpal vitality, and its intensity or permanence is associated to the pathological status of the pulp. A negative response might be associated to pulpal necrosis [6]. In this study, the pulps were vital and with a diagnosis of IP.

A disadvantage of this tool is that the trial cannot be repeated before a period of 5 minutes as results might be false. The ensued hypothermia produces anesthesia. This is the operation time of the method proposed, as it was implemented in this research [11].

Pantera et al. [11] tested if previous trial with cold temperature could affect later response to stimuli in electrical pulp tests (EPT) and found that EPT was not at all affected by the prior use of dichlorodifluoromethane as cold testing technique. They pointed out that the exposure time in cold testing is only a few seconds until the patient complains and then it is removed, and that this period is not enough to produce hypothermia-associated anesthesia [11]. For the latter to occur, temperature must drop on the level of pulpdentin union. Douglas, Malcolm and cols, evidenced that at $22^{\circ} \mathrm{C}$, A-B fibers transmission was interrupted, while that of $\mathrm{C}$ fibers was maintained. In this study, neither the clinical evaluation of the nervous fibers nor the temperatures were considered [12].

Throughout this research, we opted for direct application of cold temperature on the isolated tooth, which accounts for greater 
hypothermia than that achieved by cold testing. Said hypothermia is the reason for the success of this study. Mandibular anesthetic techniques have a failure rate of $30-80 \%$ of the cases, and $40-91 \%$ in cases of IP [13-15].

The success rate of supplementary aids for the mandibular anesthetic technique in oral infiltration of Articaine is that of $84 \%$. Intraosseus technique boosts effectiveness in 68\%. For inferior alveolar nerve block with Lidocaine, the success rate was $32 \%$ and intra-ligament injection of lidocaine had a rate of $48 \%$. [16] Articaine Infiltration was 42\% [17] and pre-medication was $40 \%$ [18]. In this study, the effective use of Cryoanesthesia was proven with a success rate of $94,4 \%$. This posts a much higher value than that obtained with the abovementioned supplementary techniques.

In this research, only age had a statistically significant difference in relation to the success or failure of the technique. It was found that starting at 25 years, probability of success increased with each additional year of age. Magalhaes et al. [19] referred to the fact that the dentin properties in its crown portion are different from those of its apical portion regarding the width and mineralization of the tubes. Wider tubes make the transmission of temperature harder In the apical portion, where dentin is mineralized and with a lower number of tubes, cold temperature is more effective. Taking this concept into account, it is possible to relate the effect of age with the success of this study. It was found that starting at 25 , success rate is higher than $80 \%$. In older patients, dentin has narrower tubes and the calcification process is more advanced, which favors the transmission of temperature [19].

Young Hak et al. [20] in 2005, while conducting a study on cryo-conservation of human teeth aiming at the creation of a future tooth bank, found enamel fractures in 4 out of 16 teeth; however, no differences were found in the hardness testing; it must be pointed out that cryo-conservation temperature should be -130 to -196 degrees. Temperatures applied on the teeth object of these study are not so extreme, as they only ranged from 9 to $23^{\circ} \mathrm{C}$. this study did not take into consideration these variables like those of Young and cols.

Further studies are needed to establish the time necessary to cool human teeth, and how long this hypothermia lasts once the cold agent has been removed, and how far the cold temperature expands beyond contact area.

\section{CONCLUSION}

Cryoanesthesia was found to be a successful technique as a supplementary aid for mandibular anesthesia in the endodontic treatment of inferior molars. It is recommended to conduct clinical tests where this technique can be compared to the conventional mandibular technique without supplementary aid.

\section{REFERENCES}

1. Silver K, Silver J (2016) The place of James Arnott (1797-1883) in the development of local anaesthesia in dentistry. Br Dent J 220(5): 249252.

2. Vera J, Ochoa J, Romero M, Vazquez-Carcaño M, Ramos-Gregorio CO, et al (2018) Intracanal cryotherapy reduces postoperative pain in teeth with symptomatic apical periodontitis: a randomized multicenter clinical trial. J Endod 44(1): 4-8.

3. Restarski JS (1946) Anesthesia induced by local refrigeration of the jaws. JADA 25(6): 433-440.

4. Martinez A (2009) Oral anesthesia: Practical guide. Pan American Medical Care, USA.

5. Malamed S (1990) Local Anesthesia Manual $3^{\text {rd }}$ (edn.) St Louis, Mosby, USA, Pp:1-332.

6. Hsiao-Wu GW, Susarla SM, White RR (2007) Use of the cold test as a measure of pulpal anesthesia during endodontic therapy: a randomized, blinded, placebo-controlled clinical trial. J Endod 33(4): 406-410.

7. Vera J, Ochoa-Rivera J, Vazquez-Carcaño M, Romero M, Arias A, et al. (2015) Effect of intracanal cryotherapy on reducing root surface temperature. J Endod 41(11): 1884-1887.

8. Gundogdu EC, Arslan H (2018) Effects of various cryotherapy applications on postoperative pain in molar teeth with symptomatic apical periodontitis: a preliminary randomized prospective clinical trial. J Endod 44(3): 349-354.

9. Yadav M, Grewal MS, Grewal S, Deshwal P (2015) Comparison of preoperative oral ketorolac on anesthetic efficacy of inferior alveolar nerve block and buccal and lingual infiltration with articaine and lidocaine in patients with irreversible pulpitis: a prospective, randomized, controlled, double-blind study. J Endod 41(11): 1773-1777.

10. Oudry E (1863) Improvement in apparatus for cooling the teeth. US Patent Office, USA.

11. Pantera EA, Anderson RW, Pantera CT (1993) Reliability of electric pulp testing after pulpal testing with dichlorodifluoromethane. J Endod 19(6): 312-314.

12. Douglas WW, Malcolm JL (1955) The effect of localized cooling on conduction in cat nerves. J Physiol 130(1): 53-71.

13. Cohen HP, Cha BY, Spångberg LS (1993) Endodontic anesthesia in mandibular molars: a clinical study. J Endod 19(7): 370-373.

14. Nusstein J, Reader A, Nist R, Beck M, Meyers WJ (1998) Anesthetic efficacy of the supplemental intraosseous injection of $2 \%$ lidocaine with 1:100,000 epinephrine in irreversible pulpitis. J Endod 24(7): 487-491.

15. Reisman D, Lector A, Nist R, Beck M, Weaver J (1997) Anesthetic efficacy of supplemental intraosseous injection of 3\% mepivacaine in irreversible pulpitis. Oral Surg Oral Med Oral Pathol Oral Radiol Endod 84(6): 676-682.

16. Kanaa MD, Whitworth JM, Meechan JG (2012) A prospective randomized trial of different supplementary local anesthetic techniques after failure of inferior alveolar nerve block in patients with irreversible pulpitis in mandibular teeth. J Endod 38(4): 421-425.

17. Fowler S, Drum M, Reader A, Beck M (2016) Anesthetic success of an inferior alveolar nerve block and supplemental articaine buccal infiltration for molars and premolars in patients with symptomatic irreversible pulpitis. J Endod 42(3): 390-392.

18. Akhlaghi NM, Hormozi B, Abbott PV, Khalilak Z (2016) Efficacy of ketorolac buccal infiltrations and inferior alveolar nerve blocks in patients with irreversible pulpitis: a prospective, double-blind, randomized clinical trial. J Endod 42(5): 691-695.

19. Magalhaes MF, Ferreira RA, Grossi PA (2008) Measurement of thermophysical properties of human dentin: effect of open porosity. J Dent 36(8): 588-594.

20. Oh YH, Che ZM, Hong JC, Lee EJ, Lee SJ, et al. (2005) Cryopreservation of human teeth for future organization of a tooth bank-a preliminary study. Cryobiol 51(3): 322-329. 